\title{
AN OPEN SOURCE WEB-GIS BASED PRECISE SATELLITE TRACKING AND VISUALISATION TOOL USING TWO LINE ELEMENT DATA
}

\author{
Abhisek Maiti ${ }^{1 *}$, Sayantan Majumdar ${ }^{1}$, Shashwat Shukla ${ }^{1}$, Shiva Reddy Koti ${ }^{1}$, Prasun Kumar Gupta ${ }^{1}$ \\ ${ }^{1}$ Geo-Informatics Department, Indian Institute of Remote Sensing, Indian Space Research Organization, Dehradun, India - (maiti, \\ s.majumdar, shukla)@ student.utwente.nl, (shivareddy, prasun)@iirs.gov.in
}

Commission V, WG V/8

KEY WORDS: Open-source, Web-GIS, Satellite tracking, Two line element, PostgreSQL, Skyfield

\begin{abstract}
:
Accurate monitoring of satellites plays a pivotal role in analysing critical mission specific parameters for estimating orbital position uncertainties. An appropriate database management system (DBMS) at the software end, could prove its potential as a convenient solution over the existing file based two line element (TLE) data structure. The current web-based satellite tracking systems, such as n2yo, satview, and satflare, are unable to provide location-based satellite monitoring. Moreover, the users need to zoom into the displayed world map for obtaining information of the satellites that are currently over the respective area. Also, satellite searching is a cumbersome task in these web-based systems. In this research work, a systematic approach has been utilised to develop a generic opensource Web-GIS based tool for addressing the aforementioned issues. This tool incorporates a PostgreSQL database for storing the parsed TLE data which are freely available on the CelesTrak (NORAD) repository. Our choice of selecting PostgreSQL as a backend DB is primarily due to its open source and scalable properties compared to other resource intensive databases. Using suitable python libraries (e.g. Skyfield and Orbit-Predictor), the position and velocity at any point of time can be accurately estimated. For this purpose, the tool has been tested on several cities for displaying location-based satellite tracking that includes different types of space-objects.
\end{abstract}

\section{INTRODUCTION}

\subsection{Advances in Satellite Tracking}

Since the past two decades, the utilization of satellite data has become an immensely important task for facilitating the monitoring of agriculture, forestry, marine and oceanography, geology, water resources and urban regional planning research domains (Citta et al., 2018; Joughin, Smith, \& Howat, 2018; Li et al., 2018; Si et al., 2018; Tangdamrongsub, Han, \& Decker, 2017). Consequently, the tracking of satellites plays a crucial role in understanding the telemetry dynamics and command networks related to the mission specific operations (Pisacane, 2005; Wertz \& Larson, 1999). In order to perform this activity, knowledge about the Keplerian orbital parameters along with the necessary sensor attributes has to be known as a priori. These elemental variables can be used to solve relativistic mathematical formulations regarding celestial mechanics associated with both effective gravitational perturbations and spatiotemporal uncertainties (Jannati, Valadan Zoej, \& Mokhtarzade, 2018; Xu, 2018).

In order to foster the utility of the perturbation in tracking the satellites, the primary governing aerospace organizations, namely North American Aerospace Defence Command (NORAD), National Aeronautics and Space Administration (NASA) and Air Force Space Command (AFSPC), jointly facilitate the applicability of the Simplified General Perturbations Satellite Orbit Model 4 (SGP4) (Kelso, 2007). In fact, this algorithm is used as a standardised satellite tracking model and has been widely accepted in both the scientific communities and the general public (Kelso, 2007; Vallado, Crawford, Hujsak, \& Kelso, 2006).

\footnotetext{
* Corresponding author
}

Currently, the two line element (TLE) data structure is the de facto standard for encoding the orbital parameters (Doornbos, Klinkrad, \& Visser, 2008; Kelso, 2007). This encoding scheme, consisting of two 70-column ASCII lines, can be subsequently processed by open source libraries like Skyfield, NASA Orbit Predictor, etc., through the underlying simplified perturbations models such as SGP4 (Kelso, 2007). This method accepts as input a NORAD TLE coupled with a specified date and time and generates the satellite location at the given epoch. In the case of real time scenario, the SPG4 procedure can be iteratively called by passing the aforementioned inputs as actual arguments. Subsequently, the raw TLE file is parsed and the values are semantically assigned to various attributes for computing the orbital state vectors consisting of latitude, longitude, and altitude components. Furthermore, the accuracy of TLE data displays a strong agreement with the precision of the SGP4 outputs (Kelso, 2007).

In essence, the TLEs, available on CelesTrak website, are also widely used as inputs for projecting the future orbital tracks of space debris pertaining to collision avoidance maneuvering, risk, close approach and forensic analysis (Carrico, Carrico, Policastri, \& Loucks, 2008). Additionally, the TLE data can be incorporated by interactive satellite trajectory visualisation tools to construct a convenient learning environment for satellite enthusiasts.

\subsection{Web-GIS based Satellite Visualisation}

In recent times, Web-GIS has been extensively utilised to build a user friendly interface for carrying out GIS related tasks such as visualisation, data analysis and contextual spatial information extraction (Fonte, Fontes, \& Cardoso, 2018; Macumber et al., 2018; Yamamoto \& Zhou, 2018). Compared to static desktop GIS, there are several advantages provided by its web-based counterpart. These include hardware independence (only the web browser is required), portability, flexibility, robustness, and 
accessibility. Moreover, it facilitates an intuitive user experience that allows a seamless and versatile platform for geographic content creation and sharing functionalities. In addition to this, it follows open geospatial consortium (OGC) specified guidelines to ensure high quality and standardized usage of application programming interfaces (APIs) (Kim \& Kim, 2002; Rainho \& Bernardino, 2018). As a result, Web-GIS could be introduced as a suitable visualisation technology in the satellite monitoring paradigm which could act as a proxy for the e-learning model (Fargher, 2018).

As of now, there exist several open source mapping libraries, spatial databases and GIS servers that broadly constitute the Web-GIS technology. Essentially, these provide feature-rich components such as interactive maps and aesthetically pleasing user experience integrated with geospatial data consistency, maintainability, increased site responsiveness, and scalability (Delipetrev, Jonoski, \& Solomatine, 2014; Steiniger \& Hunter, 2011; Tsou \& Smith, 2011; Uyaguari et al., 2018). Moreover, JavaScript mapping APIs like Leaflet and OpenLayers offer easily readable and understandable documentation that facilitates convenient code development as well as rapid deployment. Also, the recent advancements in spatial databases (PostgreSQL, Oracle Spatial, MySQL, MS SQL Server, etc.) have led to substantial performance gains on heavy network traffic management, eventually improving the robustness of the web applications (Eriksson, Berglund, \& Eriksson, 2018; Gfiting, 1994).

The popular web applications related to space object flight path studies are n2yo, satview, and satflare (n2yo, 2008; Satflare, 2011; Satview, 2008). Although these existing systems provide real time satellite pass views, the users are unable to make custom satellite search queries that cater to their corresponding requirements. Moreover, the desktop based solutions suffer from adequate portability and often require additional pre-compiled binaries, which may be cumbersome to first-time users. Hence, there is a scope for potentially improving these satellite trackers by incorporating concrete and light-weight solutions following OGC standards (Castronova, Goodall, \& Elag, 2013; Fargher, 2018; Kim \& Kim, 2002). Consequently, with the introduction of HTML5, the development of web technologies have grown exponentially, thereby massively promoting open source technologies.

In this research work, a Web-GIS based satellite tracking and visualisation tool has been developed using fully open-source libraries that aims at solving the aforementioned issues prevalent in similar applications.

\section{DATA AND METHODS}

\subsection{Existing TLE Data}

In this research, the TLE data has been utilised for determining the state of any point by considering a suitable temporal prediction model. The TLE data consist of two 70 column ASCII lines of which the first row represents the attributes of the satellite including satellite number, classification, epoch, ephemeris, etc. while the second row determines the Keplerian orbital parameters of the satellite. The specified orbital elements are inclination, eccentricity, the argument of perigee, mean anomaly, mean motion, revolution number, etc. This study is based on all available TLEs of operational satellites, GPS satellites, weather satellites, space debris and space stations.

\subsection{Methods Adopted}

Initially, the TLE data has been scraped from CelesTrack server as raw ASCII file format. This data format is a standard for distributing the list of orbital parameters of an orbiting satellite for a given epoch. Here, each set of elements are written to two 70-column ASCII lines, as described in section 2.1. The following are the steps involved in implementing the working of the proposed tool, as in Figure 1.

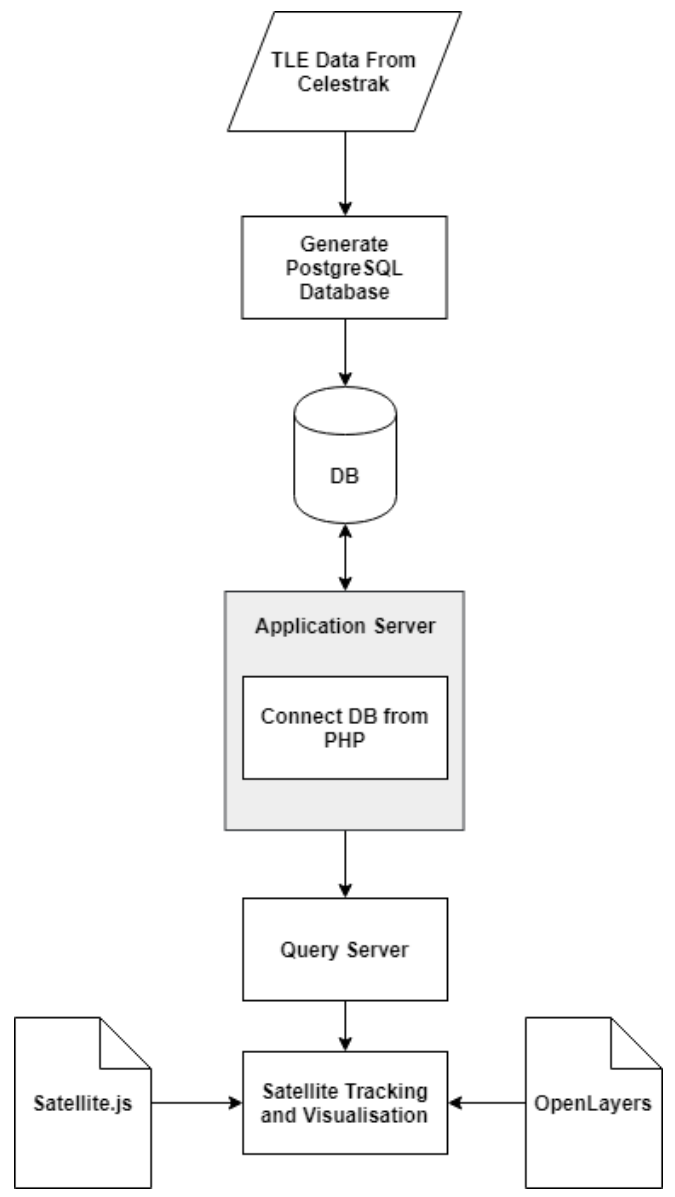

Figure 1: Schematic and Inter-process flow of the Proposed Tool.

Building the TLE Database: At the backend, the Python based Skyfield library has been used for the high precision (results agree with that of the United States Naval Observatory and their Astronomical Almanac to be within 0.0005 arc seconds) estimation of satellite trajectory, which in turn, takes the TLE data as input. However, the provided arguments have to be parsed beforehand for subsequent query processing. Currently, Python being one of the most popular and extensively used scripting languages in the space industry, a pure python based TLE parser has been developed for facilitating the application development process in a convenient and hassle-free way. The parsed TLEs are semantically placed into respective fields of the PostgreSQL database, that has been normalised up to the third normal form.

Linking with Web-GIS: In this phase of the workflow, a WebGIS environment has been developed wherein OpenLayers and Satellite.js JavaScript libraries are used to display and animate the maps respectively. The PostgreSQL DB is kept at the server side built using PHP and a suitable UI/UX has been developed. In addition to this, the TLE database is automatically updated whenever there is any change of the TLE data on the CelesTrack server. 
User Interface: The aim here is to develop a user friendly interface, wherein the respective user is able to choose the geotemporal variables such as location and time of the satellites. These are used as binding variables to a PostgreSQL query, which is then fired to display a 2D animation showing the current trajectories of selected orbiting satellites. In this case, the temporal attribute provided by the user can represent either past or future satellite orbiting scenarios. Apart from this, a provision for adding future satellite orbital information has been kept, which could be further used for trajectory simulation studies.

The entire methodology has been implemented using prototyping based Software Development Life Cycle (SDLC) approach. By following this software engineering model, the core components have been rapidly deployed. Essentially, an adequate system testing has been performed for mitigating code errors. Additionally, for software verification and validation $(\mathrm{V} \& \mathrm{~V})$, the generated outputs have been compared with similar applications.

\section{RESULTS AND ANALYSIS}

As part of verifying the functional requirements of this tool, several test cases considering different space objects and locations have been performed.

\subsection{Real-time Satellite Tracking}

In order to monitor the real time scenario for a specific satellite, the OpenLayers map update interval has been set to $500 \mathrm{~ms}$. Consequently, the scatter plot of the same appears to be a continuous curve as shown in Figure 2, wherein the current ISS position is marked by a black head. Since numerous positions are generated at a high frequency, a buffer size of maximum 2000 points have been fixed for memory efficiency. Once this upper bound of the buffer exceeds, the trailing points disappear in a sequential manner. With this robust and simple visualisation, the satellite flyby time over a particular area could be predicted. The tool also provides flexibility to the users in adjusting the time delay as per requirements. However, it has been observed that a very low time delay would cause excessively minute changes in the position, effectively leading to prolonged rendering in the visualisation process. The result of this analysis is in concordance with those of existing web-based systems.

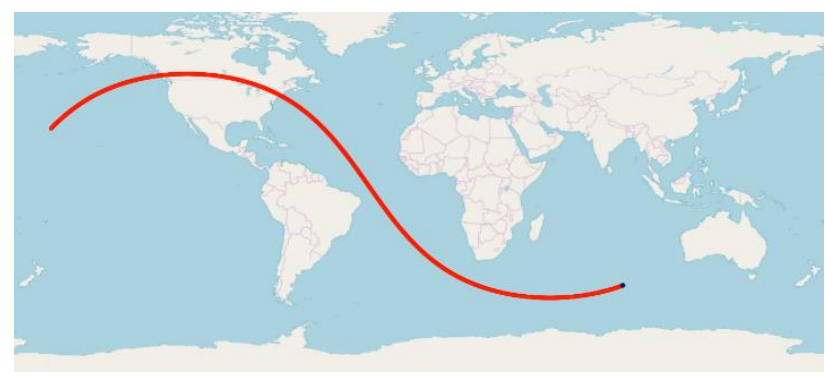

Figure 2: Real-time International Space Station (ISS) Ground Track. Black dot displays the current location while the red line shows its trajectory path.

\subsection{Simulated Analysis of Satellite Trajectories}

Another feature provided by the proposed tool incorporates a simulation environment for observing the satellite trajectories specific to a user defined time period. In this, the time delay is measured in minutes producing multiple ground tracks, as shown in Figure 3, 4 and 5. In essence, this facilitates a convenient learning environment for enthusiasts to understand the orbital patterns of the satellites in a look ahead manner - a characteristic unavailable in its real time counterpart. The trajectory of ISS exhibits an almost perfectly circular orbit traversing in a single direction around the sphere, as evident in Figure 3.

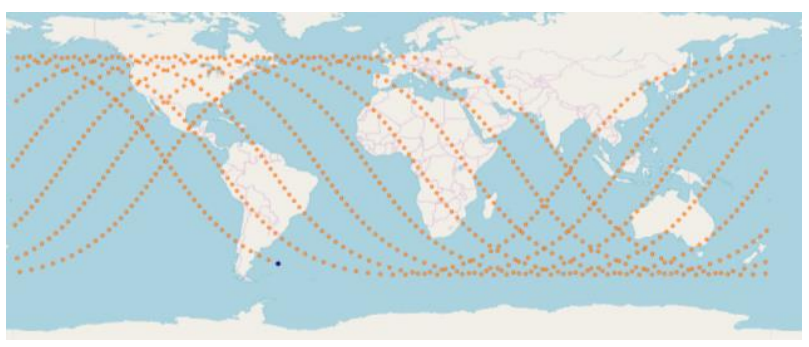

Figure 3: Simulated satellite trajectory for ISS. The comparison of this could be made with Figure 2 .

Furthermore, an attempt has been made to extend this applicability of the tool in tracking the space debris for subsequent risk analysis. Figure 4 illustrates the result of the simulated tracking of Iridium $33 \mathrm{DEB}$, wherein it has been observed that it is currently following a polar orbital trajectory.

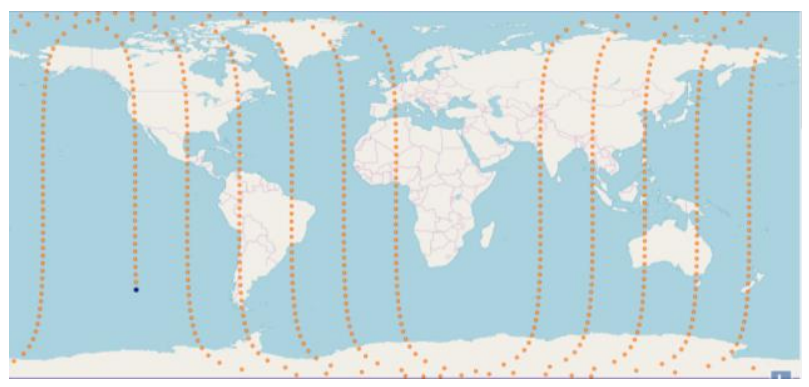

Figure 4: Results of simulated space debris tracking. In this, Iridium 33 DEB is considered as a dysfunctional space object after its collision with Cosmos 2251 in 2009 (NASA Orbital Debris Program Office, 2009).

In addition to this, the research work also involved the study of well-known resource satellites such as Worldview-4. The results obtained justify the behavioural aspects of the orbital signature (near polar) exhibited by Worldview-4, as displayed in Figure 5 . The black dot shows the last position of the satellite before screen capture whereas the other dots denote the position of the satellite in previous temporal instances.

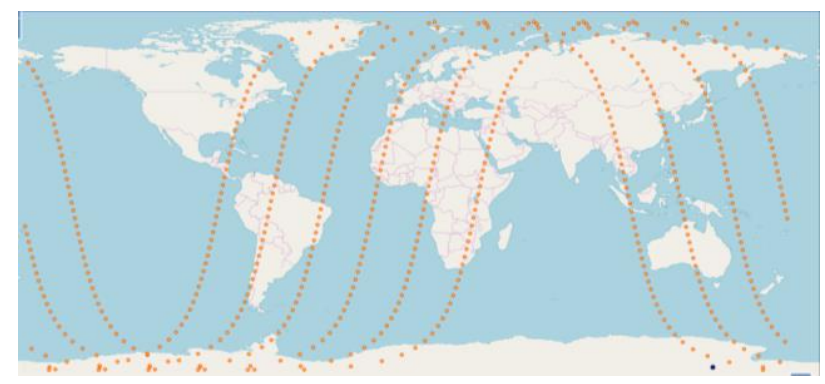

Figure 5: Simulated polar-orbiting Worldview-4 orbital trajectory.

Moreover, the tool also enables the user to visualise the geographical variations with respect to time (Figure 6). In the following graph, the latitudinal and longitudinal changes of Worldview- 4 have been computed for an overall continuous period of 8 hours, staring from 00:00 to 09:00 hrs (UTC) on $6^{\text {th }}$ 
July 2018. It can be interpreted that it readily follows the simulated trajectory (Figure 5) in which there are sharp gradient descents and steady variability for longitudes and latitudes respectively.

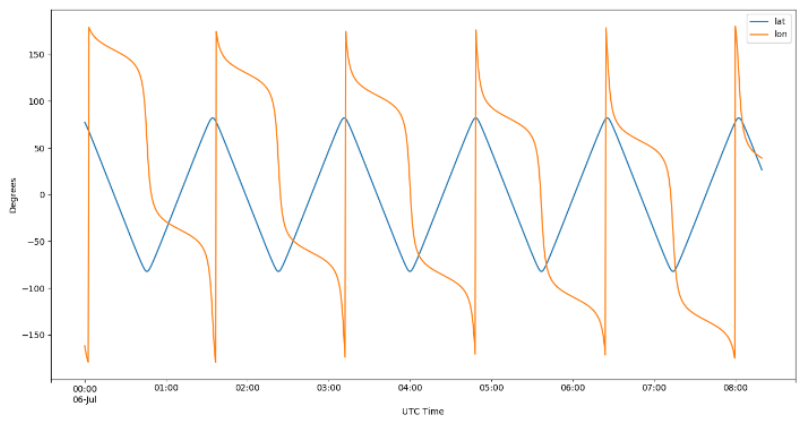

Figure 6: Temporal variations of Latitude and Longitude for Worldview-4.

\subsection{Location-based Visualisation over Different Places}

Essentially, to address the issue of location-based satellite mapping, a polar coordinate transform plot can be automatically retrieved using this tool through the Orbit-Predictor library. It is noteworthy that a limited number of satellites should be displayed for handling cluttering problems. At first, this feature has been tested for Dehradun (Figure 7 (a)) and Enschede (Figure 7 (b)) on $6^{\text {th }}$ July 2018 at 15:04 hrs CEST. These plots incorporate intuitive mapping directions with $0^{\circ}$ as north and $180^{\circ}$ as south.

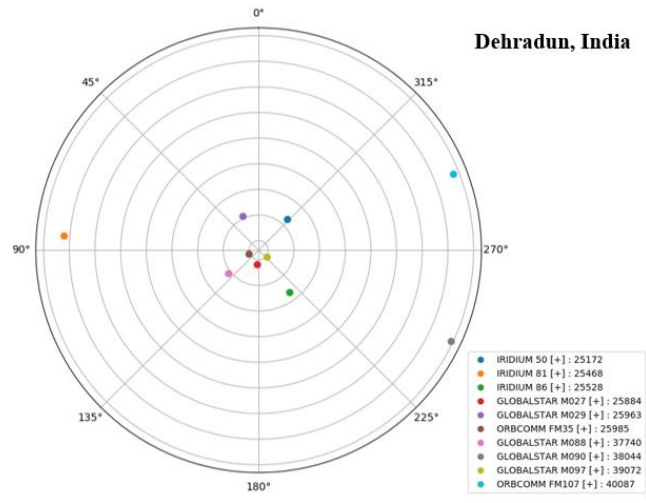

a)

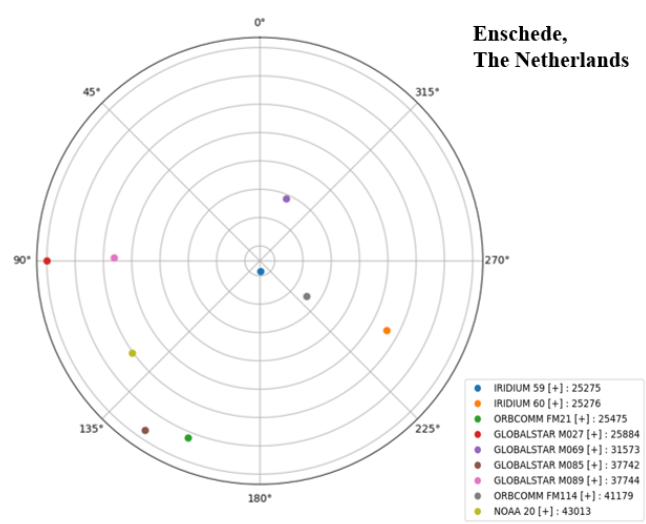

b)

Figure 7: Location-based satellite visualisation as observed in a) Dehradun, India and b) Enschede, The Netherlands.
In this case, the voluminous TLE datasets have been trimmed by adding the 'Operational' condition corresponding to the 'Status' field of the database. The matplotlib library used for evaluating these plots automatically assigns a colour ramp to the satellites denoted by the dots over the respective location. The total number of satellites observed over Dehradun are found to be higher than that of Enschede. Moreover, the study also considered the near polar regions of Svalbard (Figure 8 (a)) and Dunedin (Figure 8 (b)) as an additional test case on the same dates as above.

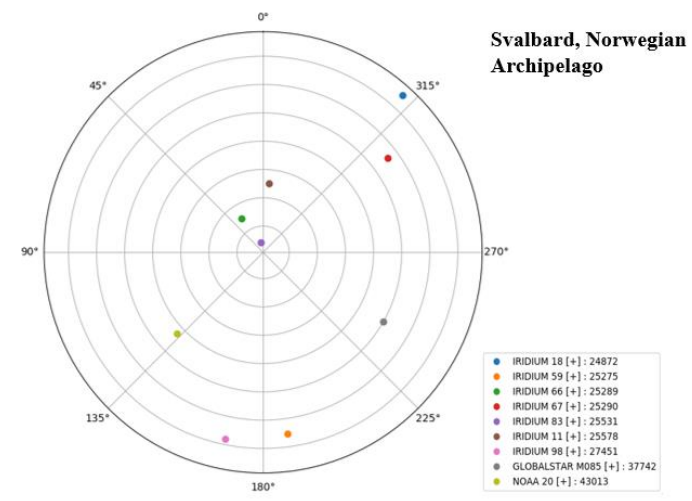

a)

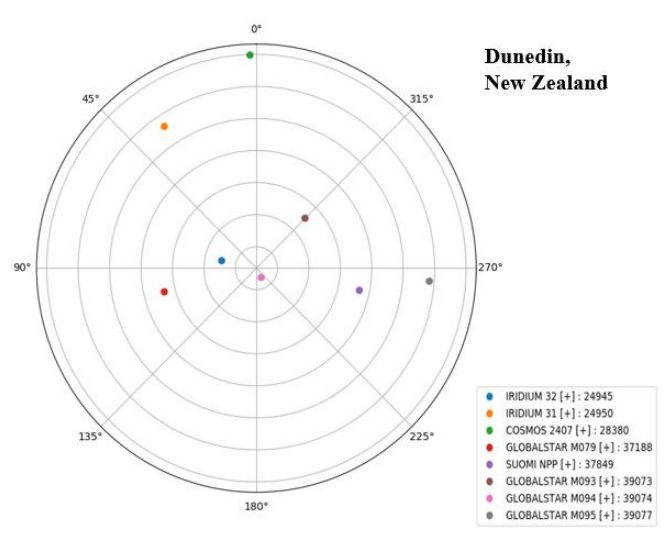

b)

Figure 8: Location-based visualisation in polar regions, a) Svalbard, Norway and b) Dunedin, New Zealand.

\section{DISCUSSION}

In case of the simulated space object tracking considering ISS, Worldview-4 and Iridium 33 space debris, it has been observed that the time delay is an important factor that controls the density of the plotted positions in the graphs. This corresponds to the continuity of the flight paths, as for example, the map refresh interval in the real time scenario is in milliseconds apparently resulting in a smooth curve whereas the simulated tracks consist of discrete positions updated in minutes. It should be noted that the displayed maps are in Plate Carree projection system.

The high inclination angle of the near polar orbiting satellites attributes to the shifting of the sub-satellite point from north to south along the projection of the Earth's axis. It could be inferred from Figure 4 and 5 that the densification of the dots in the graphs shows the locational variations. In the polar regions, the simulated positions are more spaced as compared to those of the equatorial mid latitude region. In essence, the displacement of the longitude is a function of the orbital period, i.e., the sub-satellite point shifts either to complete sunlight or darkness during the 
entire period while the satellite circles the polar regions. The sparse dots near the poles imply the lower speed of the satellite and thereby, reflects the displacement of the satellite to a low earth orbit about the poles in order to maintain a balance between the gravitational and centrifugal accelerations (Montenbruck \& Gill, 2000)

Furthermore, the tool has been developed following standard coding practices with suitable code documentation for extensibility, readability and reusability purposes. It also facilitates a semantically and succinctly designed user experience pertaining to the discussed functional requirements (in section 2).

\section{CONCLUSION AND FUTURE SCOPE}

In this preliminary study, an effort has been to promote the utility of geospatial technologies in the development of a satellite tracking tool. The uniqueness of this Web-GIS tool lies in providing the simulated ground tracks of all available satellites present in TLE format along with their respective lat/long variations and location-based satellite visualisations. Interestingly, the results showing various orbital trajectories (both real-time and simulated), exhibit high precision due to the usage of the python based Skyfield and Orbit-Predictor libraries. As part of the future software development process, the proposed tool could be ported to smartphone platforms. In order to perform a robust software $V \& V$, the output GPS satellite positions could be cross-verified with a ground based survey involving the use of DGPS or mobile GPS for obtaining orbital arrangements of the existing GPS satellites at a given instance of time. In this regard, the study strongly recommends applying 3D models for enhancing the existing 2D geo-visualisation. Also, to envisage the dynamics of space debris like Iridium 33, a convenient 3D simulation involving collision models could be incorporated in the tool as a separate library. Such features would substantially improve the user's perception about satellite trajectories wherein multiple ground tracks can be appropriately viewed. Moreover, the location-based polar plots could be used as an initial step to analyse the satellite orientations over a particular area and thereby, build suitable optimisation techniques related to satellite geometries. In conclusion, the study emphasises the practicability of this tool as an improved alternative to the existing web-based satellite tracking systems.

\section{ACKNOWLEDGEMENTS}

The authors are grateful to FOSS4G communities for promoting the free and open source libraries. They would also like to acknowledge the Indian Institute of Remote Sensing, ISRO for providing the necessary means to carry out this research work.

\section{REFERENCES}

Carrico, T., Carrico, J., Policastri, L., \& Loucks, M. (2008). Investigating orbital debris events using numerical methods with full force model orbit propagation. Advances in the Astronautical Sciences, 130(1), 407-426.

Castronova, A. M., Goodall, J. L., \& Elag, M. M. (2013). Models as web services using the Open Geospatial Consortium (OGC) Web Processing Service (WPS) Standard. Environmental Modelling and Software, 41, 72-83. https://doi.org/10.1016/j.envsoft.2012.11.010

Citta, J. J., Lowry, L. F., Quakenbush, L. T., Kelly, B. P., Fischbach, A. S., London, J. M., ... Gray, T. (2018). A multispecies synthesis of satellite telemetry data in the Pacific Arctic
(1987-2015): Overlap of marine mammal distributions and core use areas. Deep Sea Research Part II: Topical Studies in Oceanography, (xxx), $1-22$. https://doi.org/10.1016/j.dsr2.2018.02.006

Delipetrev, B., Jonoski, A., \& Solomatine, D. P. (2014). Development of a web application for water resources based on open source software. Computers and Geosciences, 62, 35-42. https://doi.org/10.1016/j.cageo.2013.09.012

Doornbos, E., Klinkrad, H., \& Visser, P. (2008). Use of two-line element data for thermosphere neutral density model calibration. Advances in Space Research, 41(7), 1115-1122. https://doi.org/10.1016/j.asr.2006.12.025

Eriksson, L., Berglund, H. E., \& Eriksson, L. (2018). Using IndexedDB with a spatial database Using IndexedDB with a spatial database.

Fargher, M. (2018). WebGIS for Geography Education: Towards a GeoCapabilities Approach. ISPRS International Journal of Geo-Information, $\quad 7(111), \quad 1-15$. https://doi.org/10.3390/ijgi7030111

Fonte, C., Fontes, D., \& Cardoso, A. (2018). A Web GIS-Based Platform to Harvest Georeferenced Data from Social Networks: Examples of Data Collection Regarding Disaster Events. International Journal of Online Engineering (IJOE), 14(2), 165172.

Gfiting, R. H. (1994). An Introduction to Spatial Database Systems. The VLDB Journal - The International Journal on Very Large Data Bases - Spatial Database Systems, 3(4), 357-399.

Jannati, M., Valadan Zoej, M. J., \& Mokhtarzade, M. (2018). A novel approach for epipolar resampling of cross-track linear pushbroom imagery using orbital parameters model. ISPRS Journal of Photogrammetry and Remote Sensing, 137, 1-14. https://doi.org/10.1016/j.isprsjprs.2018.01.008

Joughin, I., Smith, B. E., \& Howat, I. M. (2018). A complete map of Greenland ice velocity derived from satellite data collected over 20 years. Journal of Glaciology, 64(243), 1-11. https://doi.org/10.1017/jog.2017.73

Kelso, T. S. (2007). Validation of SGP4 and IS-GPS-200D Anainst GPS Ephemerides. In 17th AAS/AIAA Space Flight Mechanics Conference (pp. 427-440). Sedona, Arizona.

Kim, D., \& Kim, M. (2002). Web GIS Service Component Based On Open Environment. In IEEE International Geoscience and Remote Sensing Symposium (pp. 3346-3348). Toronto, Canada: IEEE. https://doi.org/10.1109/IGARSS.2002.1027178

Li, S., Meng, W., Liu, D., Yang, Q., Chen, L., Dai, Q., ... Li, Y. (2018). Migratory Whooper Swans Cygnus cygnus Transmit H5N1 Virus between China and Mongolia: Combination Evidence from Satellite Tracking and Phylogenetics Analysis. Scientific Reports, 8(1), 7049. https://doi.org/10.1038/s41598018-25291-1

Macumber, D., Horowitz, S., Schott, M., Nolan, K., Schiller, B., Horowitz, S., ... Schiller, B. (2018). FloorspaceJS - A New, Open Source, Web-Based Geometry Editor for Building Energy Modeling (BEM). In Simulation for Architecture and Urban Design (SimAUD) 2018 (pp. 1-8). Delft, Netherlands: National Renewable Energy Laboratory. 
Montenbruck, O., \& Gill, E. (2000). Satellite Orbits: Models, Methods and Applications. Berlin Heidelberg,: Springer-Verlag.

n2yo. (2008). N2YO. Retrieved July 4, 2018, from http://www.n2yo.com/

NASA Orbital Debris Program Office. (2009). Satellite collision leaves significant debris clouds. Orbital Debris Quarterly News (Vol. 13). https://doi.org/10.1016/0273-1177(93)90600-G

Pisacane, V. L. (Ed.). (2005). Fundamentals of Space Systems (Second Edi). New York: Oxford University Press.

Rainho, C., \& Bernardino, J. (2018). Web GIS : A new system to store spatial data using GeoJSON in MongoDB. In 13th Iberian Conference on Information Systems and Technologies (CISTI) (pp. 1-6). AISTI

Satflare. (2011). SATFLARE. Retrieved July 4, 2018, from http://www.satflare.com/

Satview. (2008). SATVIEW - Tracking Satellites. Retrieved July 4, 2018, from http://www.satview.org/

Si, Y., Xu, Y., Xu, F., Li, X., Zhang, W., Wielstra, B., ... Gong, P. (2018). Spring migration patterns, habitat use, and stopover site protection status for two declining waterfowl species wintering in China as revealed by satellite tracking. Ecology and Evolution, (January), 1-10. https://doi.org/10.1002/ece3.4174

Steiniger, S., \& Hunter, A. J. S. (2011). Free and Open Source GIS Software for Building a Spatial Data Infrastructure. In Geospatial Free and Open Source Software in the 21st Century (pp. 247-261). Berlin, Germany: Springer, Berlin.

Tangdamrongsub, N., Han, S.-C., \& Decker, M. (2017). On the use of GRACE intersatellite tracking data for improved estimation of soil moisture and groundwater in Australia. Hydrology and Earth System Sciences Discussions, (June), 1-33. https://doi.org/10.5194/hess-2017-318

Tsou, M., \& Smith, J. (2011). Free and Open Source Software for GIS education.

Uyaguari, A., Espinosa-Gallardo, E., Jácome-Guerrero, S. P., Espinel, P., Cabezas, C. F., Arias Almeida, G. I., \& Calderón, F. A. C. (2018). Open Source Web Software Architecture Components for Geographic Information Systems in the Last 5 Years: A Systematic Mapping Study. In Proceedings of the International Conference on Information Technology \& Systems (ICITS 2018) (pp. 688-699). Springer, Cham. https://doi.org/10.1007/978-3-319-73450-7_65

Vallado, D. A., Crawford, P., Hujsak, R., \& Kelso, T. S. (2006). Revisiting Spacetrack Report \#3.

Wertz, J. R., \& Larson, W. (1999). Space Mission Analysis and Design. The Space Technology Library (Third Edit). El Segundo, California: Microcosm Press and Kluwer Academic Publishers. Retrieved from http://www.amazon.ca/Space-Mission-AnalysisDesign-

James/dp/0792359011/ref=sr_1_1?s=books\&ie=UTF8\&qid=14 $30653992 \& \mathrm{sr}=1-1 \&$ keywords $=9780792359012$

Xu, P. (2018). Measurement-based perturbation theory and differential equation parameter estimation with applications to satellite gravimetry. Communications in Nonlinear Science and Numerical Simulation, 59, 515-543. https://doi.org/10.1016/j.cnsns.2017.11.021

Yamamoto, K., \& Zhou, J. (2018). Navigation System Using Web-GIS and AR for Urban Tourists. In P. Kiefer, H. Huang, N. Van de Weghe, \& M. Raubal (Eds.), 14th International Conference on Location Based Services (pp. 152-157). Zurich: ETH Zurich. https://doi.org/10.3929/ethz-b-000225609 